\title{
Surgical Treatment of Neglected Traumatic Quadriceps Tendon Rupture with Knee Ankylosis
}

\author{
Seung-Hun Lee, MD, Eun-Kyoo Song, MD, Jong-Keun Seon, MD, and Seong-Hwan Woo, MD \\ Department of Orthopedic Surgery, Center for Joint Disease, Chonnam National University Bitgoeul Hospital, Gwangju, Korea
}

\begin{abstract}
Quadriceps tendon rupture is an uncommon injury. This disabling condition is the result of direct or indirect trauma. It requires surgical repair to avoid poor outcomes in cases of neglected or chronic rupture. In most acute cases, simple tendon suture or reinsertion is suitable for an extensor mechanism reconstruction of the knee joint. However, chronic lesions often require a tendon graft or flap reconstruction. We report a case of a 15-year-old male who was diagnosed with a chronic quadriceps rupture with a patellar superior pole fracture. We performed quadriceps reconstruction using tibialis anterior allograft tendon and additional screw fixation to reconstruct the extensor mechanism and recover knee joint range of motion to prevent a high-level functional restriction. The treatment was difficult and limited due to neglect for 9-months that led to ankylosis accompanied with nonunion of tibial fracture. Our surgical treatment using allograft tendon resulted in a very good outcome after 30 months of follow-up.
\end{abstract}

Keywords: Quadriceps muscle, Ankylosis, Rupture, Reconstruction, Allograft

Quadriceps tendon rupture (QTR) is a relatively uncommon injury with an incidence of $1.37 / 100,000$ patients per year, affecting predominantly middle-aged males (male:female [4.2:1]; mean age, 51.1 years $)^{1)}$. This disabling condition is the result of direct or indirect trauma ${ }^{2,3)}$. In case of traumatic injury, indirect and violent eccentric contraction is the most frequent cause of QTR. In general, clinical history and physical examination are sufficient for the diagnosis of a QTR. The clinical diagnostic triad includes acute pain, inability to actively extend the knee, and a palpable suprapatellar gap. In case of a suspected tendon rupture, radiological investigations, such as ultrasound scan or magnetic resonance imaging, are used to confirm the diagnosis. QTR requires prompt surgical repair to avoid poor outcomes, which were pre-

Received June 18, 2015; Revised August 24, 2015;

Accepted October 5, 2015

Correspondence to: Eun-Kyoo Song, MD

Department of Orthopaedic Surgery, Chonnam National University Bitgoeul Hospital, 80 Deongnam-gil, Nam-gu, Gwangju 61748, Korea Tel: +82-62-670-9475, Fax: +82-62-670-9476

E-mail: eksong@jnu.ac.kr

This is an Open Access article distributed under the terms of the Creative Commons Attribution Non-Commercial License (http://creativecommons.org/licenses/by-nc/4.0/) which permits unrestricted non-commercial use, distribution, and reproduction in any medium, provided the original work is properly cited. viously reported in cases of neglected or chronic rupture ${ }^{4)}$.

Here, we report a case of a 15-year-old male diagnosed with a chronic quadriceps rupture with a patellar superior pole fracture of the left knee and nonunion of the left proximal tibial fracture. We performed quadriceps reconstruction using tibialis anterior allograft and additional screw fixation. At 30 months postoperatively, the range of motion (ROM) was between $5^{\circ}$ and $130^{\circ}$. This case was of interest due to its rarity and clinical importance.

The patient provided informed consent for publication of this case report and use of materials.

\section{Case Report}

A 15-year-old male patient visited our hospital with complaints of limitation of ROM in the left knee and inability to walk for 9-months. Nine months prior, he visited a local hospital after a motorcycle traffic accident and was diagnosed with an open fracture of the left femur shaft and an intra-articular fracture of the proximal tibia. He underwent an operation using the Ilizarov external fixator at the femur and tibia. Two months after the first surgery, the Ilizarov fixator was removed and changed into a monoaxial external fixator for the left femur and converted to internal fixation with a screw for the left tibia. One month later, he 
underwent an operation for conversion to internal fixation with intramedullary nailing of the left femur. At that time, he underwent manipulation of the knee joint for ankylosis. On physical examination at the initial visit to our hospital, he had tenderness and dimpling in the superior aspect of the patella and markedly decreased mobility of the patella (including mediolateral and superoinferior translation). He had quadriceps atrophy (a $3-\mathrm{cm}$ decrease in mid-thigh circumference as compared to the contralateral limb). His left knee ROM was between flexion contracture of $30^{\circ}$ and further flexion of $90^{\circ}$. On plain radiographs, nonunion of the left proximal tibial fracture and femur shaft fracture were shown. The lateral radiograph revealed a fracture in the superior pole of the patella and patella infera (Fig. 1). The ultrasound showed discontinuity of the quadriceps muscle fiber and hypoechoic hematoma (Fig. 2).

Under the presumptive diagnosis of a chronic quadriceps rupture with a patellar superior pole avulsion fracture of the left knee and nonunion of the left proximal tibia fracture, we performed quadriceps reconstruction using tibialis anterior allograft tendon and additional screw fixation. Initially, after removal of the fibrotic nonunion site, 2 cannulated screws were used to fix the nonunion fragment of the proximal tibia at full flexion of the knee (Fig. 3). The fracture site was stable enough not to need any procedure such as additional plating and bone grafting. The
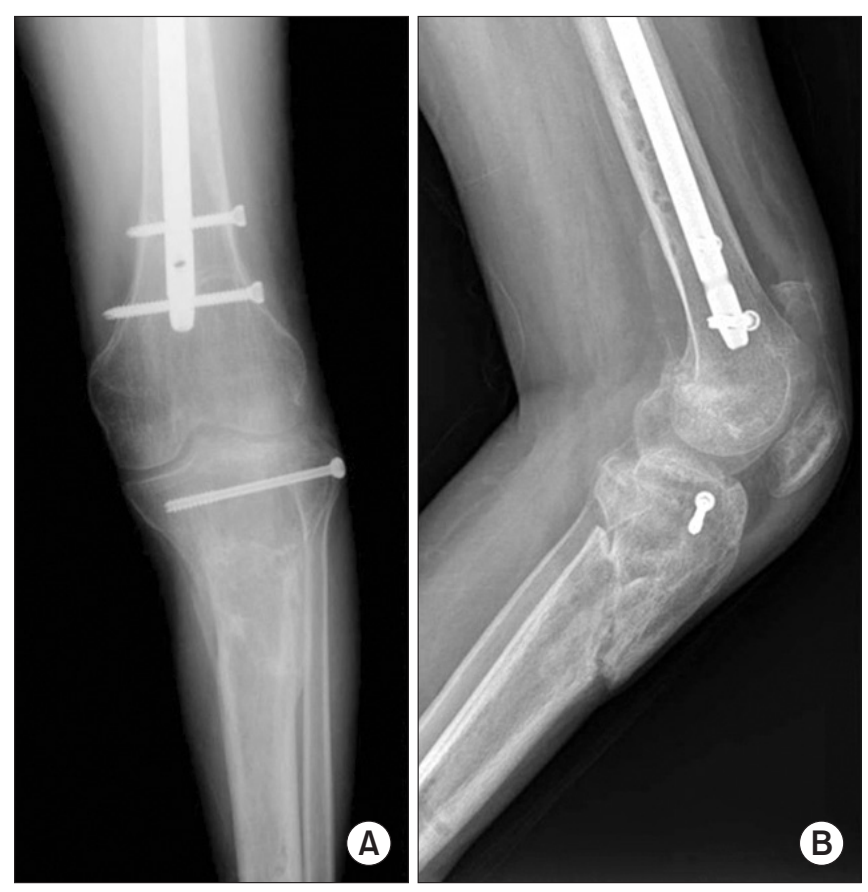

Fig. 1. (A) The anteroposterior radiograph of the left knee joint was taken at the initial visit to our hospital. (B) The lateral radiograph shows fracture of the superior pole of the patella and patellar infera. quadriceps rupture was repaired via primary end-to-end repair using heavy nonabsorbable sutures placed in running locked fashion using a Krackow technique in both the proximal and distal ends of the tendon. Suture ends are then tied together with the knee in full extension. Thus, proximal and distal ends of the ruptured quadriceps tendon was approximated with Ethibond (Ethicon, Somerville, NJ, USA) suture and additionally fixated with a roll wire to augment tendon repair. Drilling and insertion of a Steinmann pin through the patella was done and a 7-mm diameter tibialis anterior allograft was inserted through the holes. The ends of the allograft were pulled into the holes in the patella and finally augmented at the proximal end of the quadriceps tendon while the leg was in full extension (Fig. 4).

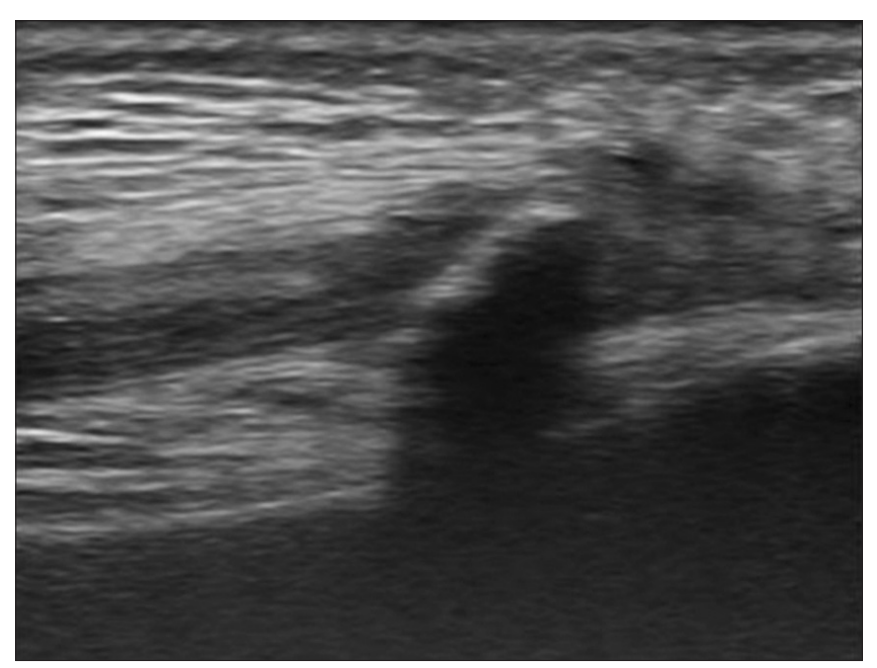

Fig. 2. Longitudinal ultrasonogram of the left anterior thigh shows discontinuity of muscle fibers and hypoechoic hematoma.
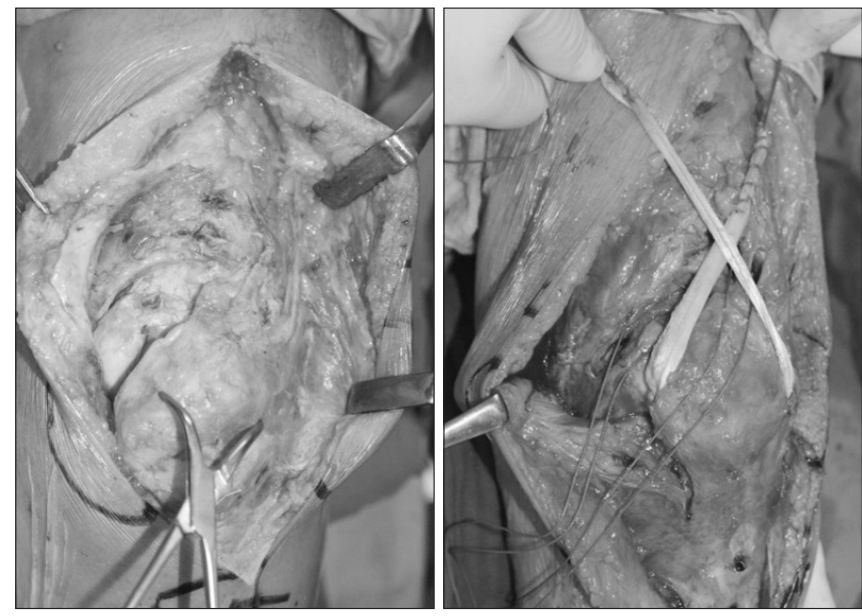

Fig. 3. The intraoperative photographs show full thickness tear of the quadriceps muscle. Quadriceps reconstruction was performed using tibialis anterior allograft tendon. 
At 3 weeks postoperatively, the patient participated in gentle ROM exercises and quadriceps and hamstring exercises were concomitantly applied. Three months after surgery, the left knee
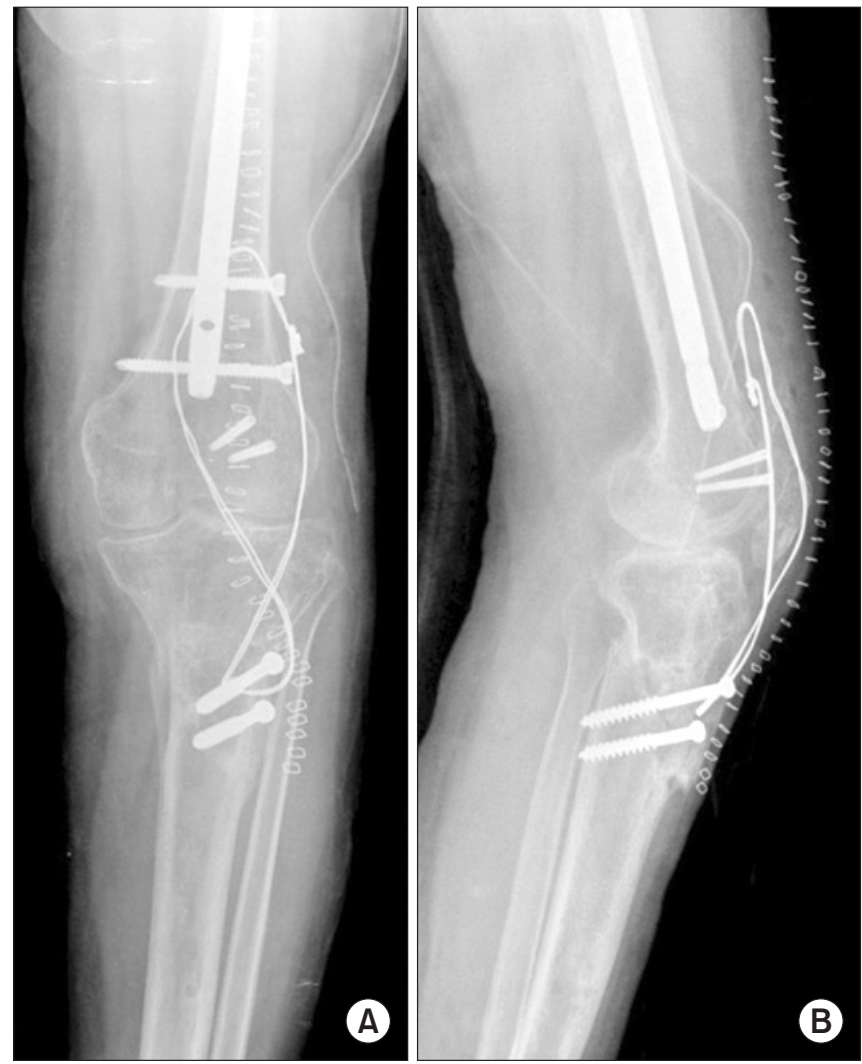

Fig. 4. The plain anteroposterior (A) and lateral (B) radiographs of the left knee were taken immediately after operation at our hospital; quadriceps reconstruction using tibialis anterior allograft tendon and additional screw fixation.
ROM was between $5^{\circ}$ of flexion and further flexion of $60^{\circ}$, and bony union was obtained. Partial weight- bearing was allowed after postoperative 5 months, and progressive improvement of knee motion was observed. At the 12-month follow-up, he could walk without aid. On the last outpatient follow-up at postoperative 30 months, the left knee ROM was between $5^{\circ}$ of flexion and further flexion of $130^{\circ}$ with persistent mild quadriceps atrophy
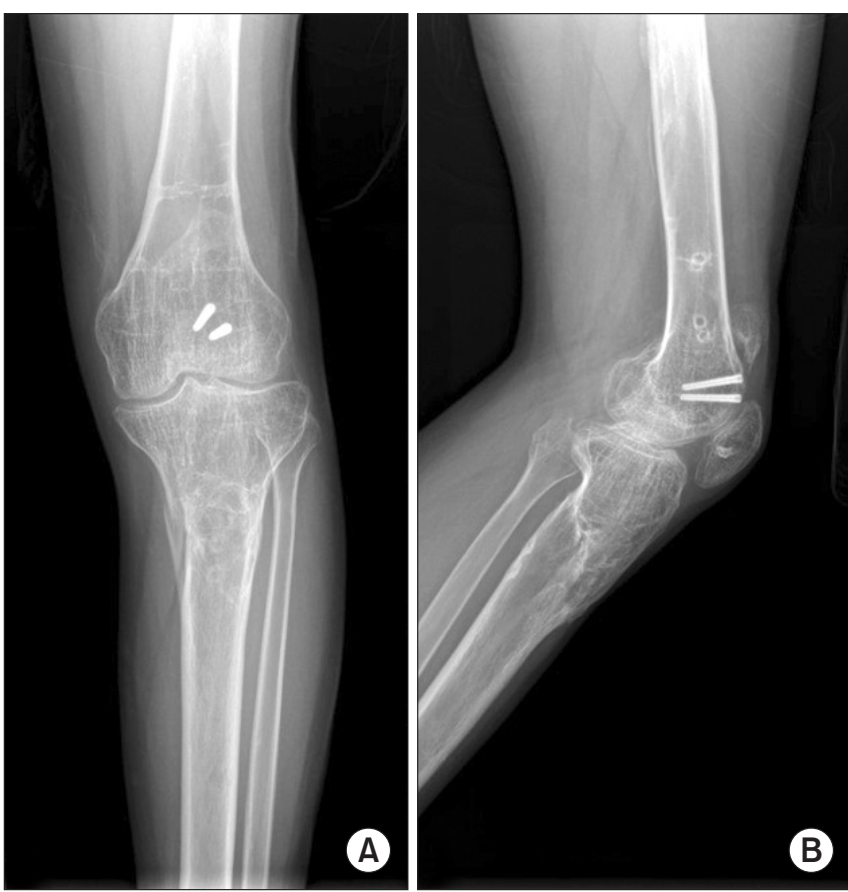

Fig. 6. The anteroposterior (A) and lateral (B) radiographs of the left knee joint taken at the last follow-up at our hospital show the metal removal state.
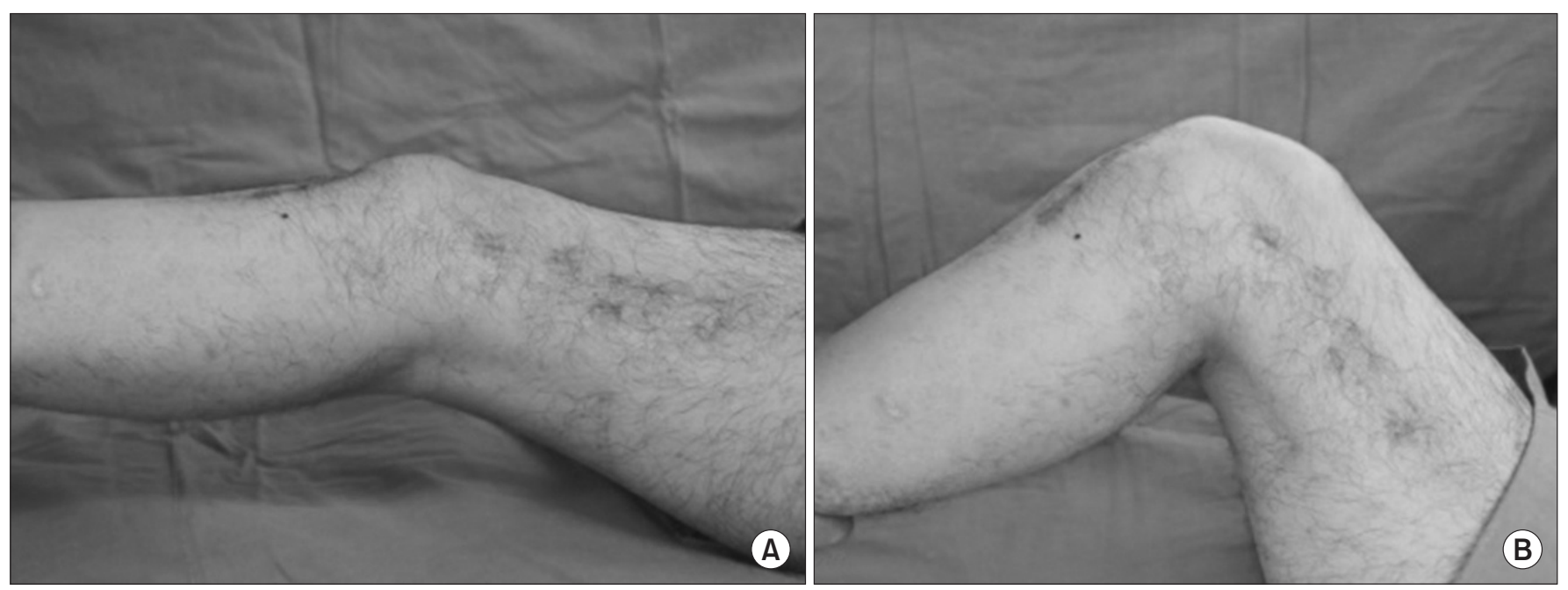

Fig. 5. The clinical photographs of the left knee joint were taken at the last follow-up at our hospital. The range of motion was from $5^{\circ}$ to $130^{\circ}$. Full extension (A) and full flexion (B) of the knee. 
and 1-cm leg length discrepancy (Figs. 5 and 6).

\section{Discussion}

Quadriceps tendon is a very strong structure contributing to the extensor mechanism of the knee. The structural and biomechanical properties of the quadriceps tendon allow it to sustain very high loads without rupture ${ }^{5}$. In most acute cases, only simple tendon suture or reinsertion is suitable for an extensor mechanism reconstruction of the knee joint. However, chronic lesions often require a tendon graft or flap reconstruction ${ }^{6}$. Extensor mechanism injuries involving the loss of the ability to stretch the knee, such as QTRs and patella fractures, have an incidence of $0.5 \%$ to $6 \%^{5,6}$. The most common sites of tears are localized between 1 and $2 \mathrm{~cm}$ to the superior pole of the patella ${ }^{7)}$. QTR accompanied with an avulsion fracture of the patellar superior pole is very rare, when passive ROM is applied after long-term immobilization following the major trauma as in our case. Augmentation techniques of lengthening quadriceps tendon and repair are required because significant retraction of quadriceps tendon is observed in such cases of delayed surgery ${ }^{6,8)}$.

In our patient, the knee ROM was limited during the period of application of an external fixator for the femoral and tibial fractures. In addition, 4 weeks of immobilization with a cylinder splint after change to intramedullary nailing led to a decrease in postoperative patellofemoral joint motion, limitation of the knee ROM, and weakness of quadriceps tendon. Hence, he received knee manipulation, which is considered to have led to avulsion fracture on the superior pole of the patella.

We performed surgical treatment on this 15 -year-old patient to reconstruct the extensor mechanism and mediate recovery of the knee joint ROM to prevent a high-level functional restriction and associated diminished quality of life. The treatment was very difficult and limited due to neglect for 9-months that resulted in knee ankylosis with nonunion of the tibial fracture.

In this patient, the surgical treatment using allograft tendon resulted in a very good outcome after 30-month follow-up. Several reconstruction options have been reported including delayed augmented repair, external fixation, the use of synthetic materials, autograft, and allograft tissues. Nazarian and Booth" reported on 36 patients treated with a fresh frozen whole extensor mechanism allograft including quadriceps tendon, patella, patella tendon, and tibial tubercle. At an average of 3.6 years of follow-up, 34 patients were noted to have a successful result. Using allograft tissues provides the advantage of avoiding donor site morbidity and allows implantation of a large graft. However, the use of allograft tissue carries some risk of transmission of infectious disease and there can be biologic incorporation ${ }^{10)}$.

In conclusions, the neglected traumatic QTR with knee ankylosis, including the patellar superior pole avulsion fracture, is a very rare and difficult condition with limited therapeutic options. Our quadriceps reconstruction using tibialis anterior allograft tendon resulted in a successful outcome and satisfactory knee joint function after 30-months of follow-up.

\section{Conflict of Interest}

No potential conflict of interest relevant to this article was reported.

\section{References}

1. Clayton RA, Court-Brown CM. The epidemiology of musculoskeletal tendinous and ligamentous injuries. Injury. 2008;39:1338-44.

2. Rueda MA, Halley WL, Gilchrist MD. Fall and injury incidence rates of jockeys while racing in Ireland, France and Britain. Injury. 2010;41:533-9.

3. Chiu WT, Chiang YH, Hung KS, Tsai SH, Lin JW. The injury epidemiology transition in South Asia. Injury. 2010;41:975-6.

4. Rasul AT Jr, Fischer DA. Primary repair of quadriceps tendon ruptures: results of treatment. Clin Orthop Relat Res. 1993;(289):205-7.

5. Konrath GA, Chen D, Lock T, Goitz HT, Watson JT, Moed BR, D’Ambrosio G. Outcomes following repair of quadriceps tendon ruptures. J Orthop Trauma. 1998;12:273-9.

6. Rougraff BT, Reeck CC, Essenmacher J. Complete quadriceps tendon ruptures. Orthopedics. 1996;19:509-14.

7. Hak DJ, Sanchez A, Trobisch P. Quadriceps tendon injuries. Orthopedics. 2010;33:40-6.

8. Volk WR, Yagnik GP, Uribe JW. Complications in brief: Quadriceps and patellar tendon tears. Clin Orthop Relat Res. 2014;472:1050-7.

9. Nazarian DG, Booth RE Jr. Extensor mechanism allografts in total knee arthroplasty. Clin Orthop Relat Res. 1999;(367): 123-9.

10. Druskin SC, Rodeo SA. Novel treatment of a failed quadriceps tendon repair in a diabetic patient using a patellaquadriceps tendon allograft. HSS J. 2013;9:195-9. 